\title{
O ensino de vigilância à saúde do trabalhador no Curso de Enfermagem
}

\author{
TEACHING SURVEILLANCE TO WORKERS' HEALTH IN NURSING COURSES \\ LA ENSEÑANZA DE LA VIGILANCIAA LA SALUD DEL TRABAJADOR EN EL CURSO \\ DE ENFERMERÍA
}

\section{Maria Silvia Monteiro', Edmar Vieira dos Santos ${ }^{2}$, Lilian Shizue Kawakami ${ }^{3}$, Marie Wada ${ }^{4}$}

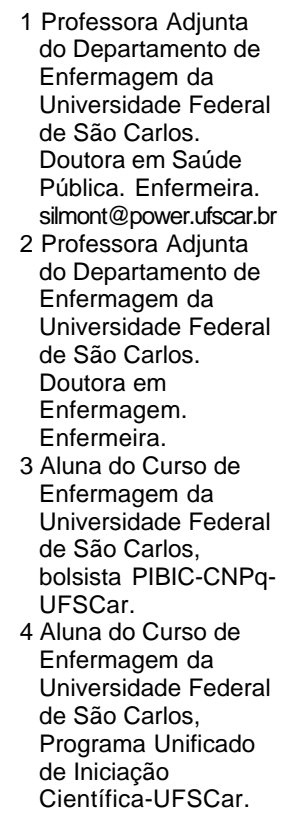

\begin{abstract}
RESUMO
Este estudo tem como objetivo descrever a experiência de ensino de vigilância à saúde do trabalhador, desenvolvida na disciplina obrigatória de Enfermagem na Saúde do Trabalhador no Curso de Graduação em Enfermagem da Universidade Federal de São Carlos. Como estratégia de ensino foi desenvolvida a análise epidemiológica de todas as Comunicações de Acidentes de Trabalho registradas junto ao Instituto Nacional de Seguridade Social, agência São Carlos, referentes ao município de São Carlos e ao ano de 2000. Foram desenvolvidos conteúdos relativos à vigilância à Saúde do Trabalhador e temas correlacionados. Com base no sistema de informações construído, foram realizadas pelos alunos atividades práticas relativas à sistematização das informações e à utilização do software empregado para inserção, consolidação e análise dos dados. São discutidos os resultados obtidos pelos alunos com as atividades realizadas, as dificuldades apresentadas e as possibilidades de utilização dessas estratégias para o ensino.
\end{abstract}

\section{DESCRITORES}

Educação em enfermagem.

Saúde do trabalhador.

Acidentes de trabalho.

Epidemiologia.

\section{ABSTRACT}

The objective of this study is to describe the experience at the Federal University of São Carlos concerning the mandatory discipline Nursing and Workers' Health, taught at the undergraduate course in Nursing, which focuses on the activities that concern workers' health surveillance. As a teaching strategy, an epidemiological analysis of all labor accidents registered at the local agency of the social security system referring to the municipality of São Carlos, State of São Paulo, in the year of 2000 was carried out. Based on this information system the students developed practical activities related to the organization of the data and the use of the software to insert, consolidate and analyze the information. Besides this, themes concerning workers' health surveillance and related issues have been developed. The results obtained in these activities have been evaluated and are discussed in this article.

\section{KEYW ORDS}

Education nursing.

Occupational health.

Accidents, occupational. Epidemiology.

\section{RESUMEN}

Este trabajo tiene como objetivo describir la experiencia de enseñanza de vigilancia a la salud del trabajador dentro de la disciplina obligatoria de Enfermería del Trabajador en el Curso de Graduación en Enfermería de la Universidad Federal de São Carlos. Como estrategia de educación en enfermería se desarrolló el análisis epdemiológica de las Comunicaciones de Accidentes de Trabajo registradas junto al Instituto de la Seguridad Social, agencia São Carlos, referentes al Municipio de São Carlos, y al año de 2000. Fueron desarrollados contenidos relativos a la vigilancia de la Salud del Trabajador y temas correlacionados. A partir del sistema de informaciones construido fueron realizadas por los alumnos actividades prácticas relacionadas a la sistematización de las informaciones y a la utilización de software empleado para inserción, consolidación y análisis de los datos. Son discutidos los resultados obtenidos por los alumnos con las actividades realizadas, las dificultades presentadas y las posibilidades de utilización de estrategias para la educación.

\section{DESCRIPTORES}

Educación en enfermería. Salud laboral.

Accidentes de trabajo. Epidemiología. 


\section{INTRODUÇÃO}

No Brasil a legislação relativa à saúde e trabalho nas empresas só foi regulamentada na década de 70 em decorrência da grande incidência de acidentes de trabalho, que chegou a atingir $18 \%$ da mão-de-obra segurada ${ }^{(1)}$. Esta estabeleceu a obrigatoriedade do desenvolvimento de ações relativas à segurança e à medicina do trabalho, através da criação de serviços especializados nesta área.

Já a legislação referente ao desenvolvimento de ações relativas à saúde e trabalho no Sistema Público de Saúde só foi concretizada na década de 90, porém alguns municípios já desenvolviam noa anos oitenta Programas de Saúde do Trabalhador na Rede Pública, como por exemplo as experiências de São Paulo e Bauru ${ }^{(2-3)}$.

Na década de 90 ocorreu a criação do Sistema Único de Saúde-SUS, o qual contemplou, como uma de suas áreas de atuação, a de Saúde do Trabalhador, definida como

um conjunto de atividades que se destina, através das ações de vigilância epidemiológica e sanitária, à promoção e proteção da saúde dos trabalhadores, assim como visa à recuperação e reabilitação da saúde dos trabalhadores submetidos aos riscos e agravos advindos das condições de trabalho...(4).

O desenvolvimento de ações de saúde do trabalhador pelos Programas de Saúde do Trabalhador na rede pública, principalmente no estado de São Paulo, gerou a necessidade de construir um arcabouço legal para dar suporte a estas ações e permitir a sua consolidação no SUS. No ano de 1998 duas portarias foram editadas com este objetivo, a primeira aprovando a Instrução Normativa de Vigilância em Saúde do Trabalhador e a segunda estabelecendo procedimentos para orientar e instrumentalizar as ações e serviços de saúde do trabalhador no SUS $^{(5-6)}$.

A Vigilância à Saúde do Trabalhador foi conceituada como

uma atuação contínua e sistemática, ao longo do tempo, no sentido de detectar, conhecer, pesquisar e analisar os fatores determinantes e condicionantes dos agravos à saúde relacionados aos processos e ambientes de trabalho, em seus aspectos tecnológico, social, organizacional e epidemiológico, com a finalidade de planejar, executar e avaliar intervenções sobre esses aspectos, de forma a eliminá-los ou controlá-los ${ }^{(5)}$.

Em 2002 uma nova portaria foi criada com o objetivo de estruturar a rede nacional de atenção integral à saúde do trabalhador $^{(7)}$.

O Departamento de Enfermagem da Universidade Federal de São Carlos oferece para os 30 alunos do curso de graduação em enfermagem uma disciplina obrigatória de Enfermagem na Saúde do Trabalhador, a qual desde 1993 aborda conteúdos relativos à saúde do trabalhador no SUS.

Como estratégia de ensino para ministrar o tema de vigilância à saúde do trabalhador foram desenvolvidos conteúdos a ela relativos ou correlacionados e também a análise epidemiológica das Comunicações de Acidentes de Trabalho (CAT) registradas junto ao Instituto Nacional de Seguro Social, agência São Carlos, referentes ao Município de São Carlos, e ao ano de $2000(\mathrm{n}=852)$.

A partir do sistema de informações construído foram realizadas pelos alunos atividades práticas relacionadas à sistematização das informações e à utilização do software empregado para inserção, consolidação e análise dos dados.

A escolha da CAT como fonte de informação baseou-se no fato de que esta é uma das poucas fontes de dados sobre saúde do trabalhador no país. Apesar de ter por finalidade a notificação do acidente com o objetivo de que o acidentado possa ter acesso ao benefício previdenciário, o conjunto delas no Brasil constitui o maior banco de dados na área de Saúde do Trabalhador.

As limitações relativas à utilização das comunicações de acidentes de trabalho incluem a sua restrição por abrangerem somente os trabalhadores cobertos pelo seguro acidentário da Previdência Social (INSS) e os acidentes efetivamente notificados, excluindo funcionários estatutários, trabalhadores do setor informal, autônomos e empregadas domésticas ${ }^{(8)}$. E também o sub-registro de acidentes ${ }^{(9)}$, além da precariedade no preenchimento das comunicações, que causa perda de informações.

Outro aspecto a ser destacado é o da importância de se estudar os eventos registrados nas CATs, os acidentes de trabalho, tendo em vista que estes são fenômenos "socialmente determinados, previsíveis e preveníveis"(10) , e constituem uma forma de violência por poderem causar a morte ou a invalidez permanente de jovens e adultos. Deste modo representam importante causa de morbidade, incapacidade e mortalidade entre trabalhadores.

No Brasil no ano de 2003 o coeficiente de acidentes de trabalho foi de 1.7 e o coeficiente de morbidade de 10.89. Foram registrados 18.510 casos de doenças, para uma população de 22.980.911 trabalhadores ${ }^{(11)}$.

Em trabalho de revisão de achados epidemiológicos em estudos de acidentes do trabalho, analisando o período entre 1994 e 2004, identificou-se que a incidência acumulativa anual variou de 2.0 em 1996 para 1.5 em 2000, tomando por base os registros de acidentes de trabalho típicos e de trajeto ${ }^{(12)}$.

O mesmo estudo, ao analisar trabalhos que tinham por objeto pesquisas de base populacional (que incluem todos os trabalhadores registrados ou não) apontou para uma incidência cumulativa anual de acidentes de trabalho variando entre $3 \%$ e $5 \%$.

As publicações sobre enfermagem e saúde do trabalhador tem privilegiado a análise da exposição dos trabalhadores de enfermagem aos riscos à saúde no trabalho ${ }^{(13-15)}$, diferentemente deste artigo, que aborda o ensino de saúde do trabalhador na formação do enfermeiro. 
Os conhecimentos da área de saúde do trabalhador tornam-se no momento atual ainda mais necessários na formação dos enfermeiros frente ao aumento da duração na vida no trabalho, o qual se deve ao envelhecimento da população que tem como consequência o aumento da exposição aos riscos à saúde no trabalho, tendo como um dos resultados a ocorrência de acidentes de trabalho.

$\mathrm{O}$ aumento da expectativa de vida ao nascer e a redução da taxa de fecundidade levaram a mudanças importantes na composição da população por faixa etária ${ }^{(16-19)}$. Este processo já vem ocorrendo há mais tempo nos países desenvolvidos o que levou a restauração, prevenção e promoção da capacidade para o trabalho através do desenvolvimento de estudos e programas centrados na capacidade para o trabalho dos trabalhadores em envelhecimento ${ }^{(20)}$.

Também nos países em desenvolvimento vêm ocorrendo mudanças na legislação previdenciária que levam à extensão da duração da vida no trabalho. No ano de 2003, no Brasil, a mudança ocorrida na legislação previdenciária do setor público aumentou a idade mínima de aposentadoria para 55 anos para mulheres e 60 anos para homens, além da exigência de 30 anos de contribuição ao sistema previdenciário.

A importância do tema vigilância em saúde do trabalhador como subsídio para o desenvolvimento de ações de saúde do trabalhador no SUS impõe a necessidade de inclusão de conteúdos sobre a temática na formação do enfermeiro, inclusive através do desenvolvimento de habilidades na construção e no manejo de sistemas de informação.

Este estudo tem por objetivo descrever as atividades de ensino relativas à capacitação do enfermeiro para a atuação no desenvolvimento de ações de vigilancia à saúde do trabalhador, através da utilização de um sistema de informações baseado nas CATs.

\section{MÉTODO}

A fonte de dados foi constituída por todos os registros de acidentes do trabalho $(\mathrm{n}=852)$ relativos ao município de São Carlos e referentes ao ano de 2000, através das cópias das Comunicações de Acidentes de Trabalho encaminhadas ao INSS. Os dados existentes nas comunicações foram codificados e transcritos para uma planilha. Em seguida, foram revisados para alimentação do sistema de informação. Este foi construído utilizando-se o programa Epi-info versão 6.04d do Center for Diseases Control and Prevention, Atlanta, Georgia, USA.

A variável estudada relativa à empresa, o ramo de atividade da empresa em que ocorreu o acidente, foi codificada utilizando-se a Classificação Nacional de Atividade Econômica - CNAE ${ }^{(21)}$. As variáveis relativas ao acidentado foram: a idade, o sexo e a ocupação, segundo a Classificação Brasileira de Ocupações - $\mathrm{CBO}^{(22)}$. As relativas ao acidente incluíram: o tipo e a causa, a causa externa, a natureza e a localização da lesão, o tempo decorrido entre o início da jornada e o acidente, a duração prevista de tratamento, o local de atendimento e o mês de ocorrência.

Para codificar os dados relativos ao acidente foi utilizada a Classificação Internacional de Doenças - CID 10 para as variáveis "natureza da lesão" e "causa externa da lesão"(23). Para a variável "causa do acidente" foi utilizada a "Codificação do Agente Diretamente Envolvido na Produção da Lesão" e para a variável "localização da lesão" a "Codificação da Lesão Segundo a Localização", ambos adaptados a partir do manual do SISCAT $^{(24)}$. A identidade dos acidentados foi tratada de maneira a preserva-lhes o sigilo.

A construção deste sistema de informações permitiu realizar a análise descritiva das comunicações de acidentes de trabalho registradas no INSS relativas ao ano de 2000 e ao município de São Carlos. Esta possibilitou identificar: o ramo de atividade da empresa em que ocorreu o acidente; um perfil do acidentado segundo a faixa etária, o gênero, a ocupação, a natureza da lesão, a sua causa externa e a localização da lesão, o tipo e a causa do acidente, o mês de sua ocorrência, a duração provável do afastamento, o regime de tratamento e o tempo decorrido entre o início da jornada e o acidente.

\section{DISCUSSÃO}

Em relação ao desenvolvimento das atividades de ensino, o conteúdo abordado incluiu os temas: epidemiologia aplicada à saúde do trabalhador, acidentes de trabalho, investigação de acidentes de trabalho, saúde do trabalhador no SUS e vigilância à saúde do trabalhador e teve duração de dez horas de aulas teóricas e oito horas de aulas práticas, conduzidas por duas professoras efetivas responsáveis pela disciplina para 30 alunos.

No desenvolvimento das atividades de ensino contou-se com dois monitores, alunas do curso de graduação em enfermagem, que participaram da construção do sistema de informação de acidentes de trabalho através do desenvolvimento de projetos de iniciação científica.

Foram utilizadas duas salas de aula, uma comum e a outra equipada com microcomputadores, nos quais foram instaladas cópias do programa Epi-info. 
O conhecimento prévio dos alunos sobre informática foi levantado, tendo sido identificando que $57.7 \%$ deles haviam frequientado cursos sobre o tema. Somente $3.9 \%$ dos alunos referiram ter realizado curso de Epi-info.

As atividades práticas desenvolvidas foram a codificação, a inserção e a análise dos dados. Para a codificação dos dados utilizou-se o seguinte material: Ficha de Codificação de Acidentes de Trabalho, com o respectivo Manual de Preenchimento, padronizado de acordo com o estudo realizado no Município de São Carlos; Fichas de Comunicações de Acidentes de Trabalho registrados, referentes aos diversos setores da economia e aos tipos de acidentes, selecionadas para codificação e para leitura (para garantir o sigilo, foram apagados os dados de identificação da empresa, do trabalhador e das testemunhas do acidente); Classificação Nacional de Atividade Econômica - CNAE, referente ao ramo de atividade da empresa em que ocorreu o acidente ${ }^{(14)}$; Classificação Brasileira de Ocupações - CBO, referente à ocupação ${ }^{(15)}$; Classificação Internacional de Doenças - CID 10 para a variável "natureza da lesão"(16); para "causa externa da lesão" o "Cap. XX: Lesões, Envenenamento e Algumas Outras Consequiências de Causas Externas"; Manual de Codificação do Agente Diretamente Envolvido na Produção da Lesão e de Codificação da Lesão, segundo a localização, adaptados do manual do SISCAT ${ }^{(17)}$, para as variáveis "causa do acidente" e "localização da lesão",

Para o desenvolvimento da atividade de codificação a turma de alunos foi orientada a organizar-se em duplas e para cada uma delas foi entregue uma pasta. Os professores explicaram o conteúdo das pastas, das fontes de referência e o procedimento para preenchimento das planilhas com os dados (variáveis) a serem destacados das CATs.

Os exemplares das fontes de referência circularam entre as duplas conforme foram requisitadas. Sob orientação dos professores, os alunos foram preenchendo as planilhas com os dados das CATs selecionadas para esse fim e, em seguida, passavam à leitura de outro bloco de CATs, selecionados com o objetivo de ampliar a sua percepção sobre as diferentes características dos acidentes. Ao final, foi feita uma apreciação da atividade no que se refere às facilidades, dificuldades e oportunidades de aprendizagens propiciadas.

\section{REFERÊNCIAS}

1. Costa JLR. Evolução histórica da medicina do trabalho. Cad CEBES. 1981;1(2):1-6.

2. Costa DF, Carmo JC, Settini MM, Santos VP, organizadores. Programa de Saúde dos Trabalhadores: a experiência da Zona Norte: uma alternativa de saúde pública. São Paulo: Hucitec; 1989.
A atividade de inserção dos dados foi feita utilizando: um roteiro de orientação para inserção e análise dos dados e um roteiro contendo os códigos registrados nas CATs do setor a ser analisado. Essa atividade foi desenvolvida em grupos de dez alunos, sendo que cada um deles utilizou um equipamento de informática. Primeiramente, o professor realizou uma apresentação do programa Epi-info e de seus recursos para a inserção e a análise dos dados. As informações transcritas para as planilhas foram inseridas pelos alunos simulando a construção de um sistema de informação. Em seguida, os dados inseridos eram revisados pelos colegas e os erros mais comuns e as estratégias para evitá-los eram apresentados e discutidos.

Para a análise dos dados foi utilizado um roteiro de orientação. Com este em mãos, o arquivo do sistema de informação acessível no equipamento de informática e seguindo as instruções do roteiro, os alunos elaboraram tabelas e gráficos sobre os acidentes de trabalho registrados relativos às instituições que desenvolvem ações de saúde no município de São Carlos-SP.

Fizeram também uma análise individual dos resultados. Em seguida, foi realizada uma discussão sobre os resultados obtidos pelos alunos, comparando-se as semelhanças e diferenças dos resultados por eles encontrados com os de outros estudos que utilizaram as CATs como fonte de dados ou que foram desenvolvidos em instituições públicas de saúde.

\section{CONCLUSÃO}

Esta experiência de ensino mostrou que ao desenvolverem as atividades os alunos apresentaram diferentes graus de dificuldade na realização das tarefas propostas.

Apesar do número de alunos por grupo ser de apenas dez indivíduos, houve pouca tolerância por parte deles em relação aos colegas com menos habilidades no uso da informática.

A avaliação das atividades de codificação, inserção e análise dos dados desenvolvidas pelos alunos foi considerada muito boa ou ótima por cerca de $80 \%$ deles; $71.5 \%$ consideraram a experiência adquirida no conteúdo sobre vigilância à saúde do trabalhador da disciplina como muito boa ou ótima.
3. Pimenta AL, Capistrano Filho D, organizadores. Saúde do trabalhador. São Paulo: Hucitec; 1988.

4. Gouveia R. Saúde pública, suprema lei: a nova legislação para a conquista da saúde. São Paulo: Mandacaru; 2000. 
5. Brasil. Ministério da Saúde. Portaria n. 3.120, de $1^{\circ}$ de julho de 1998. Aprova a instrução normativa de vigilância em saúde do trabalhador no SUS. Diário Oficial da União, Brasília, $1^{\circ}$ jul. 1998. Seção 1, p. 28-30.

6. Brasil. Ministério da Saúde. Portaria n. 3.908 de 30 de outubro de 1998. Estabelece procedimentos para orientar e instrumentalizar as ações e serviços de saúde do trabalhador no Sistema Único de Saúde [legislação na Internet]. Brasília; 1998. [citado 2007 abr. 9]. Disponível em: http://www.rnsites.com.br/P3908MS.doc

7. Brasil. Portaria n. ${ }^{\circ} 1.679$ de 19 de setembro de 2002. Dispõe sobre a estruturação da rede nacional de atenção integral à saúde do trabalhador no SUS e dá outras providências [legislação na Internet]. Brasília; 2002. [citado 2007 abr. 9]. Disponível em: http://www.anamt.org.br/portaria1679.html

8. Binder MCP, Almeida IM, Wludarski SL. Estudo dos acidentes de trabalho registrados pela Previdência Social no período de 1995-1999, em Botucatu, São Paulo. Cad Saúde Pública. 2001; 17(4):915-24.

9. Binder MCP, Cordeiro R. Sub-registro de acidentes do trabalho em localidade do estado de São Paulo, 1997. Rev Saúde Pública. 2003;37(4):409-16.

10. Binder MCP, Almeida IM. Investigação de acidentes de trabalho. São Paulo: FUNDACENTRO; 2000. Combate aos acidentes fatais; p. 35-51.

11. Brasil. Ministério do Trabalho e Emprego. Estatísticas de acidentes de trabalho - 2003 [texto na Internet]. Brasília; 2003. [citado 2006 mar. 23]. Disponível em: http://www.mte.gov.br/ Empregador/segsau/estatisticas/acidentes/estatistica/2003/

12. Santana V, Nobre L, Valdvogel BC. Acidentes de trabalho no Brasil entre 1994 e 2004: uma revisão. Ciênc Saúde Coletiva. 2005;10(4):841-55.

13. Alexandre NMC, Moreira Filho DC. Dores nas costas e enfermagem. Rev Esc Enferm USP. 1996;30(2):267-85.

14. Pafaro RC, De Martino MMF. Estudo do estresse do enfermeiro com dupla jornada de trabalho em um hospital de oncologia pediátrica de Campinas. Rev Esc Enferm USP 2004;38(2):152-60.
15. Alexandre NMC, Moraes MAA, Corrêa Filho HR. Evaluation of a program to reduce back pain in nursing personnel. Rev Saúde Pública. 2001;35(4):755-60.

16. Monteiro MS. Envelhecimento e capacidade para o trabalho entre trabalhadores brasileiros [tese]. São Paulo: Faculdade de Saúde Pública, Universidade de São Paulo; 1999.

17. Monteiro MS, Gomes JR, Ilmarinen J, Korhonen O. Ageing and work ability among Brazilian workers. In: Goedhard WJA, editor. Ageing and work 4: healthy and productive aging of older employees. The Hague: ICOH Scientific Committee on Aging and Work; 2000. p. 68-71.

18. Monteiro MS, Ilmarinen J, Gomes JR. Work ability, health sickness absence of Brazilian female workers in a research centre by age group. In: Ilmarinen J, Lehtinen S. editors. Past, present and future of work ability: proceedings of the $1^{\text {st }}$ International Symposium on Work Ability. Helsinki: Finnish Institute of Occupational Health; 2004. p. 60-70. (People and Work - Research Reports 65).

19. Ilmarinen J. Aging workers. Occup Environ Med. 2001;58(8):546-52.

20. Ilmarinen J. Aging workers in the European Union-Status and promotion of work ability, employability and employment. Helsinki: Finnish Institute of Occupational Health, Ministry of Social Affairs and Health, Ministry of Labour; 1999.

21. Brasil. Ministério do Trabalho. Manual de orientação Rais: Relação Anual de Informações Sociais. Brasília; 1997. Anexo III - Classificação Nacional de Atividades Econômicas (CNAE); p. $115-24$.

22. Brasil. Ministério do Trabalho. Manual de orientação Rais: Relação Anual de Informações Sociais. Brasília; 1997. Anexo I Classificação Brasileira de Ocupações (CBO); p. 41-83.

23. Organização Mundial da Saúde (OMS). CID - 10: classificação estatística internacional de doenças e problemas relacionados à saúde. 10ª rev. São Paulo: EDUSP; 1996.

24. Binder MCP. Manuais de codificação: anexos 2 e 3 adaptado do SISCAT. Botucatu: Faculdade de Medicina de Botucatu/ UNESP; 1998. 